\title{
Vieillesse: liberté de mourir ou soins négligés
}

\section{Hans Kurt}

Dr méd., ancien président de la Société suisse de psychiatrie et psychothérapie

C'était une belle mort. Une de ces morts qui donnent confiance en la vie (Alain Rebetez) - De nombreuses personnes craignent de devenir une charge (Werner Schärer, Pro Senectute) - Les coûts de la santé pour les seniors augmentent de 4 milliards («Tages-Anzeiger») - Les Suisses veulent choisir l'heure de leur mort («Tribune de Genève») - L'euthanasie d'une Britannique en bonne santé à Bâle fait polémique (RTS) - Choisir de mourir à 104 ans: le scientifique australien David Goodall était venu à Bâle afin d'avoir recours aux services d'une organisation suisse d'aide au suicide... Il espérait que la médiatisation de son cas puisse influencer le débat sur l'euthanasie dans son pays («Le Temps»).

L'aide au suicide fait régulièrement les gros titres. En Suisse alémanique, une campagne publicitaire mettant en scène des personnalités alimente le débat. EXIT, la principale organisation d'assistance au décès de Suisse, compte 110000 membres. Le corps médical se retrouve au cœur de l'actualité avec des formules toutes faites telles que «traitement arbitraire» ou «industrie lucrative de la maladie profitant des personnes en fin de vie». Bien souvent, les exigences politiques s'amalgament avec des considérations éthiques et les médecins risquent de devenir des exécutants de désirs parfois défendus avec une ardeur de missionnaire.

\section{Liberté de mourir: conditions et problématique}

Selon les directives de l'Académie suisse des sciences médicales (ASSM) de 2013, qui ont été reprises dans le Code de déontologie de la FMH, «l'assistance au suicide ne fait pas partie de l'activité médicale, car elle est contraire aux buts de la médecine». C'est à chaque médecin de décider s'il accepte ou refuse d'apporter son aide et de vérifier que les exigences autorisant l'assistance au décès sont réunies: le patient souffre d'une maladie grave à l'issue fatale, il est capable de discernement, son désir de mourir est mûrement réfléchi, persistant et ne résulte pas d'une pression extérieure, différentes alternatives thérapeutiques ont été proposées ou exécutées.
L'évaluation de la capacité de discernement et des possibilités thérapeutiques requiert des compétences médicales, notamment en psychiatrie, même si certains le contestent. Que l'on songe ici aux dépressions insuffi-

C'est à chaque médecin de décider s'il accepte ou refuse d'apporter son aide.

samment diagnostiquées des personnes âgées, chez qui le désir de mourir peut être un symptôme de la maladie [1]. Cette appréciation devient compliquée si l'on tient compte des nouvelles directives de l'ASSM, publiées en 2018. Les limitations fonctionnelles du patient lui causant une souffrance insupportable s'ajoutent désormais aux symptômes de la maladie. Or, comme ni les limitations fonctionnelles ni la souffrance insupportable ne sont définies plus en détail, ces deux critères sont difficiles à évaluer. Cet élargissement du patient en fin de vie à des facteurs psychosociaux imprécis constitue un nouveau défi pour les médecins et la société en général.

En ce qui concerne la hausse des frais des soins et de la prise en charge chez les personnes âgées - une place en institution peut vite coûter plus de 10000 francs par mois -, l'exigence "son désir de mourir [...] ne résulte pas d'une pression extérieure» pose également problème. Les personnes âgées ne sont pas seulement des individus autodéterminés, mais aussi des membres d'une famille et de la société. Des termes ou énoncés tels que «suicide altruiste» ou «société encourageant les aînés à mettre fin à leurs jours» doivent unique- 
ment servir de pistes pour poursuivre la discussion [2] La question de savoir si toutes les organisations d'assistance au décès exercent leur activité de manière altruiste reste ouverte.

\section{Aide au suicide - quelques chiffres}

En 2014, l'Office fédéral de la santé publique a enregistré 742 cas de suicide assisté dans la population domiciliée en Suisse, ce qui correspond à 1,2\% des décès. Par rapport à l'année précédente, cela représente une hausse de $26 \%$. Les femmes ont davantage tendance à solliciter une aide au suicide que les hommes. Pendant la période 2010 à 2014, 94\% des personnes qui y ont recouru avaient 55 ans ou plus et souffraient en majorité d'une maladie mortelle. Ces chiffres n'englobent pas les nombreuses personnes qui se rendent en Suisse depuis l'étranger pour accomplir un suicide assisté, ni les cas non déclarés, c'est-à-dire les actions suicidaires indirectes telles que les comportements à risque ou les actes de renoncement. En comparant les taux de suicide avec le nombre de suicides assistés, on constate que le taux de suicide diminue après 2008, alors que le nombre de suicides assistés augmente, en particulier chez les aînés. Cela laisse supposer qu'un groupe croissant de personnes âgées qui se seraient de toute façon suicidées choisissent l'option du suicide assisté.

\section{En 2014, l'Office fédéral de la santé publique a enregistré 742 cas de suicide assisté dans la population domiciliée en Suisse.}

Une étude épidémiologique approfondie [3] présente des chiffres qui soulèvent la discussion. Entre 2003 et 2008, tous les suicides assistés déclarés par les organisations d'assistance au décès ont été enregistrés. Pendant les cinq années qu'a duré l'enquête, 1301 personnes se sont donné la mort de cette façon; 862 avaient entre 65 et 94 ans, alors que les 439 autres, soit un tiers des décès, avaient moins de 65 ans. Si dans $84 \%$ des cas (N 1093), il a été possible de prouver que les critères autorisant un suicide assisté étaient remplis, aucune raison valable telle que le diagnostic d'une maladie grave ou l'issue fatale n'a pu être trouvée pour les $16 \%$ restants. Les données sur les diagnostics et les maladies ayant entraîné une assistance au décès révèlent que la plupart des cas enregistrés de 1998 à 2009 étaient motivés par des cancers, suivis par les maladies neurodégénératives. Un cinquième des cas, soit un groupe relativement important, était cependant dû à d'autres causes, qui restent inconnues.
Une étude néerlandaise datant de 2010 indique que 60\% de la population interrogée pourraient envisager de recourir à une aide au suicide, mais que seuls 2,7\% des personnes en fin de vie l'ont fait. Quant au corps médical, si plus de deux tiers (78\%) des médecins ayant participé à une enquête de l'ASSM (2014) approuvent l'aide au suicide, moins de la moitié seraient prêts à la pratiquer.

\section{Quelques points de discussion}

Jusqu'à aujourd'hui, le Conseil fédéral a refusé de réglementer explicitement l'aide au suicide par une loi. Il invoque les dispositions du droit pénal en vigueur, qui prévoient notamment que l'aide au suicide ne constitue pas un acte punissable lorsque la personne prêtant

\section{Le droit à l'autodétermination équivaut-il à un droit à l'aide au suicide?}

assistance n'est pas poussée par un mobile égoïste (art. 115 du Code pénal suisse, CP). Le meurtre sur demande de la victime est par contre punissable selon l'art. 114 du Code pénal. Comme en Suisse l'aide au suicide est presque exclusivement proposée par des organisations, on peut cependant se demander s'il ne faudrait pas au moins que leurs activités soient réglées au niveau fédéral.

De plus, faut-il obligatoirement qu'une organisation prête son assistance ou ne pourrait-on pas envisager des solutions individuelles? Cette question est également débattue. Pour le moment, les organisations d'assistance au décès ne peuvent pas pratiquer leur activité dans les établissements médico-sociaux (EMS), les hôpitaux ou les cliniques. Ainsi, un pensionnaire d'EMS désireux de mourir doit généralement être transféré ailleurs pour recevoir une aide au suicide. Vu que le suicide assisté et la prise en compte des souhaits des pensionnaires d'EMS et des patients font toujours plus débat, des discussions sont actuellement menées à différents niveaux sur l'opportunité d'ouvrir les institutions aux organisations d'assistance au décès sous certaines conditions.

La liberté de mourir réclamée par les organisations d'assistance au décès suscite des réactions contrastées et souvent émotionnelles. Une personne âgée en bonne santé, qui ne souffre donc d'aucune maladie et ne présente aucun signe d'une mort prochaine, doitelle avoir le droit d'accomplir un suicide assisté de sa propre volonté? Le droit à l'autodétermination équivaut-il à un droit à l'aide au suicide? Est-ce qu'«en avoir assez de la vie» est une raison suffisante pour mettre fin à ses jours avec de l'aide? Les nouvelles directives de l'ASSM vont dans ce sens. Mais répond-on ici à une ten- 
dance générale, et le corps médical, en particulier, veut-il participer à une telle évolution?

Depuis longtemps, le droit des personnes atteintes de troubles psychiques de bénéficier d'une aide au suicide suscite de nombreuses controverses en psychiatrie. Si les malades psychiques se voient refuser ce droit, cela en-

\section{La réaction de l'entourage d'une personne ayant recouru à l'aide au suicide n'a pas encore été abordée ici.}

traîne une inégalité de traitement par rapport aux personnes souffrant de maladies physiques et contribue à l'exclusion et à la stigmatisation. Cependant, il est souvent difficile d'apprécier si le désir de suicide résulte d'une décision mûrement réfléchie ou d'un état mental altéré.

\section{En guise de conclusion}

Dans les pays voisins, la Suisse est bien connue pour son ouverture face au suicide assisté. Mais cela ne signifie en aucun cas que l'aide au suicide et les questions qui lui sont liées, comme les conditions-cadres ou les aspects organisationnels, éthiques et juridiques, soient résolues et incontestées.

La réaction de l'entourage d'une personne ayant recouru à l'aide au suicide n'a pas encore été abordée ici. Une étude [4] a montré que les proches souffrent fréquemment de trouble de stress post-traumatique et de dépression après le suicide assisté d'un membre de la famille.

Le droit à une fin de vie autodéterminée n'est pas controversé. Mais notre existence ne présuppose-t-elle pas également un droit à recevoir des soins? Une société progressiste n'est pas une société dans laquelle le suicide devient une évidence, mais une société qui offre suffisamment de soins, de traitements et d'attention
(«Der Bund», 11.6.2012). Que proposer d'autre en réponse à un désir de suicide? Mentionnons ici des approches telles que les soins palliatifs, le mouvement des hospices ainsi que les offres à bas seuil et hors murs de la gériatrie ou de la psychiatrie gériatrique.

Pour terminer, sans financement adéquat et suffisant des soins et de l'accompagnement des personnes âgées ou souffrant de maladies chroniques, celles-ci risquent de ne plus vouloir être à la charge des autres et de commettre un suicide dit altruiste. On parle alors d'une société qui encourage le suicide des aînés, autrement dit qui est contente lorsque ceux-ci mettent fin à leurs jours le moment venu.

Des représentations idéalistes comme «une belle mort", «mourir en bonne santé», "c'est à moi de choisir quand je veux partir» soulagent peu les patients, les soignants et les médecins dans leur quotidien. Le débat sur l'aide au suicide doit rester ouvert. Il est nécessaire, car il nous empêche d'utiliser des formules toutes faites et de suivre des tendances sans nous soucier des conséquences.

\section{Bibliographie}

1 Minder J, Ajdacic-Gross V et Hepp U. Suicide de la personne âgée. Swiss Medical Forum. 2018;18(10):230-5.

2 Wedler H. Selbstbestimmtes Sterben: nur eine Utopie? Psychother im Alter. 2013;11:11-26.

3 Steck N, Junker C, Maessen M, et al. Suicide assisted by right-to-die associations: a population based cohorty study. Intern J Epidem. 2014;1-9.

4 Wagner B, Müller J et Maercker A. Death by request in Switzerland: Posttraumtic stress disorder and complicated grief after witnessing assisted suicide. Europ Psychiat. 2012;27(7):542-6.

\section{Prises de position}

Prise de position du comité IPSILON, avril 2017, http://www.ipsilon.ch/ fr/actuel/news.cfm

Prise de position de la SPPA - Suicide assisté pour les personnes âgées, novembre 2014, https://www.sgap-sppa.ch/fr/specialistes/publications-specialisees

Directives de l'ASSM - Attitude face à la fin de vie et à la mort, juin 2018, https://www.samw.ch/fr/Publications/Directives.html 